\title{
MicroRNA-4651 targets bromodomain-containing protein 4 to inhibit non-small cell lung cancer cell growth
}

Jiangnan Zheng

First Affiliated Hospital of Soochow University

Lingyun Dong

Nantong University

Yan Zhang

Jiangsu University

Shang Cai

Soochow University

Xiaoyun Hu

Nantong University

Min-bin Chen

Jiangsu University

Ye-han Zhu ( $\nabla$ zhuyhhxzz@163.com )

First Affiliated Hospital of Soochow University

Research

Keywords: miR-4651, BRD4, NSCLC, cell proliferation

Posted Date: December 6th, 2019

DOI: https://doi.org/10.21203/rs.2.18318/v2

License: (c) (i) This work is licensed under a Creative Commons Attribution 4.0 International License.

Read Full License 


\section{Abstract}

Background: Bromodomain-containing protein 4 (BRD4) overexpression in non-small cell lung cancer (NSCLC) is important for cancer cell progression. The aim of the present study is to silence BRD4 through expression of its targeted microRNAs in NSCLC cells.

Methods: Expression of BRD4 and its targeting miRNA, microRNA-4651 (miR-4651), was tested by qPCR and Western blotting assays. Genetic strategies were utilized to exogenously alter miR-4651 expression. NSCLC cell growth, proliferation and migration were tested.

Results: miR-4651 selectively targets and negatively regulates BRD4 in A549 and primary human NSCLC cells. The Ago-2 immunoprecipitation experiments further confirmed that miR-4651 directly binds to BRD4 mRNA. Significantly, in A549 cells and primary NSCLC cells ectopic overexpression of miR-4651 downregulated BRD4's 3-UTR activity and its expression, both were however elevated with miR-4651 inhibition. Functional studies demonstrated that NSCLC cell growth, proliferation and migration were significantly inhibited with miR-4651 overexpression, but enhanced with miR-4651 inhibition. BRD4 reexpression, by an 3'-UTR mutant BRD4, reversed miR-4651 overexpression-induced inhibitions on A549 cells. Additionally, miR-4651 overexpression or inhibition failed to affect the functions of BRD4-KO A549 cells. In vivo, miR-4651-overexpressed A549 xenografts grew significantly slower than control A549 xenografts in severe combined immunodeficient mice. At last we show that miR-4651 is downregulated in human NSCLC tissues, correlating with BRD4 elevation.

Conclusions: miR-4651 targets BRD4 to inhibit NSCLC cell growth in vitro and in vivo.

\section{Background}

Lung cancer has become one leading cause of cancer-related human mortalities globally, with estimated over two million new cases and 1.75 million deaths in 2018 [1]. Non-small cell lung cancer (NSCLC) is the primary type of lung cancer, accounts for over $80 \%$ of all lung cancers [1, 2]. In the clinical practices, surgical resection is one preferred therapy for NSCLC [2-4]. Yet the high recurrent rate as well as the relatively resistant to adjuvant chemotherapy/targeted therapies preclude the surgical treatment as the curative option in the fast majority of NSCLC patients [2-4]. Therefore, there is an urgent need to further explore the underlying pathological mechanisms for NSCLC progression, and to develop possible novel targeted strategies $[3,5]$.

Bromodomain-containing protein 4 (BRD4), the most-studied and one primary BET (bromodomain and extraterminal) family protein, contains two $\mathrm{N}$-terminal bromodomains recognizing the acetylated lysine residues [6]. Published literatures have demonstrated that BRD4 binds directly to acetylated histones and transcription factors, and recruits positives transcription elongation factor $b$ ( $P$-TEFb) and possible other transcriptional regulators [6]. BRD4 is overexpressed in NSCLC, correlating with the poor prognosis and other clinical features of NSCLC patients [7,8]. Studies have proposed that BRD4 is a major contributor to the invasive phenotype of NSCLC, represents as an important therapeutic oncotarget $[7,8]$. It is critical for 
the translation and expression of several key oncogenes, including c-Myc, Bcl-2 and cyclin D1 [6, 9-11]. Inhibition or silencing of BRD4 can potently inhibit NSCLC cell progression in vitro and in vivo $[7,8]$.

MicroRNAs (miRNAs) are a large family of conserved, short and single-strand noncoding RNAs (ncRNAs), with 21-25 nucleotides long [12-15]. By sequence-specific binding to the 3'-UTR (untranslated region) of targeted mRNA, miRNA could suppress mRNA translation and/or induce mRNA degradation, thereby regulating gene expression at the post-transcriptional level [12-15]. In cancer cells miRNAs could exert regulatory effects to almost all cancerous behaviors, including cell proliferation, cell cycle progression, apoptosis, metastasis and epithelial-mesenchymal transition (EMT) $[16,17]$. Dysregulation of miRNA has been commonly detected in NSCLC and other lung cancers, plays a pivotal role in cancer formation, tumorigenesis, progression, therapy-resistance and prognosis predication [16, 18]. Ulivi et al., revealed that miR-328 is significantly higher in NSCLC patients than that in the healthy donors, represents a potential diagnostic biomarker of NSCLC, especially for early-stage tumors [19]. Similarly the study by Wang et al., demonstrated that miR-21 expression is higher in NSCLC patients' serum samples, which has significant prognostic significance [20]. Previous functional literatures have shown that miR-152 negatively regulated ADAM17 and possible other targets to suppress NSCLC cell progression [21, 22].

Studies have suggested that expression of BRD4-targeting miRNAs could be a novel and efficient strategy to inhibit human cancer cells with BRD4 overexpression [23-25]. The results of this study indentified microRNA-4651 (miR-4651) as a novel BRD4-targeting miRNA, suppressing NSCLC cell progression in vitro and in vivo.

\section{Materials And Methods}

Chemicals and reagents. The antibodies utilized in this study were purchased from Abcam (Cambridge, $M A)$. Fetal bovine serum (FBS), DMEM, RMPI and all other cell culture reagents were purchased from Hyclone (Logan, UT). Puromycin, polybrene, and all other chemicals were provided by Sigma-Aldrich Chemicals Co. (St. Louis, Mo). All primers, sequences, viral constructs and expression vectors were designed, provided and sequence verified by Shanghai Genechem Co. (Shanghai, China), unless otherwise mentioned. Annexin V, propidium iodide (PI), the reagents for PCR assays and transfection assays were obtained from Thermo-Fisher Invitrogen (Shanghai, China).

A549 cell culture. A549 NSCLC cell line was purchased from Shanghai Institute of Biochemistry and Cell Biology (Shanghai, China), cultured in DMEM together with 10\% FBS and necessary antibiotics. Cells were maintained at $37^{\circ} \mathrm{C}$ in a humidified $\mathrm{CO}_{2}$ incubator. Cells were routinely subjected to mycoplasma/microbial contamination examination every 3-6 months. STR profiling, population doubling time, and morphology were always checked to verify the genotype.

Primary human cells and tissues. The primary human lung cancer cells, derived from three writteninformed consent NSCLC patients (male, 53 to 59-year old, stage-III), were provided by Dr. Jiang [26, 27]. The primary cells were cultured in RPMI medium with necessary supplements and antibiotics [26, 27]. 
From ten written-informed consent primary NSCLC patients (all male, 46 to 64-year old, NSCLC, stage-III, all administrated at authors' institutions), the fresh lung cancer tissues and paired surrounding lung epithelial tissues were obtained, stored in liquid nitrogen and subjected to further biomedical analyses. No patients were treated with any additional therapies before surgery. The protocols of using human cells and tissues were approved by the Ethics Committee of Soochow University, in according to Declaration of Helsinki.

Quantitative real-time PCR (qPCR). Total RNA was extracted from cultured cells or human tissues by the TRIzol reagents, with RNA concentration determined with the NanoDrop ND-1000 spectrophotometer (NanoDrop Technologies, Wilmington, DE). Total RNA was converted into complementary DNA (cDNA), which was subjected to qPCR analyses using a SYBR Premix Ex Taq ${ }^{\text {TM }}$ Kit (TaKaRa, Tokyo, Japan). GAPDH was tested as the reference gene and internal control for testing BRD4 and its target genes. Expression of miR-4651 was tested through the TaqMan microRNA qPCR assay kit, using U6 as the internal control. qPCR quantification was through $2^{-\Delta C t}$ method using the following formula: $2^{-(C t}$ of target gene-Ct of reference gene). The RNA primers of this study were listed in Table-1.

BRD4 luciferase reporter assay. As previously described [28], the BRD4's 3'-UTR sequence containing the predicted miR-4651 binding sites (at position 1715-1722) was amplified by Shanghai Genechem Co. (Shanghai, China), which was sub-cloned into the PMIR-REPORT miRNA Expression Reporter vector (Ambion; Thermo Fisher Scientific, Shanghai, China), establishing the luciferase reporter plasmid, pMIRBRD4-3》-UTR. The latter was transfected to NSCLC cells using the Lipofectamine 2000 protocol. Cells were then subjected to applied genetic modifications, with luciferase activities detected at $48 \mathrm{~h}$ by the Dual-Luciferase Reporter assay system (Promega).

Ectopic miR-4651 overexpression or inhibition. The pri-miR-4651 sequence and its anti-sense sequence were synthesized and sequence-verified by Shanghai Genechem Co. (Shanghai, China), individually subcloned into the GV369 lentiviral construct (Shanghai Genechem Co.). The construct was transfected to HEK-293T cells, together with the lentivirus-packing helper plasmids, establishing the pri-miR-4651 expression lentivirus ("LV-pri-miR-4651") and the pri-miR-4651 anti-sense expression lentivirus ("LVantagomiR-4651"). The virus was added to A549 cells or primary human NSCLC cells (cultured in the polybrene-containing medium). Puromycin $(2.5 \mu \mathrm{g} / \mathrm{mL})$ was always added to select stable cells (for $10-$ 12 days), with miR-4651 expression tested by qPCR.

Transfection of miR-4651 mimic. NSCLC cells were initially seeded into the six-well tissue-culturing plates $\left(2 \times 10^{5}\right.$ cells per well). Lipofectamine 2000 protocol was carried out for the transfection of $500 \mathrm{nM}$ of the wild-type ("WT") or the mutant ("Mut") miR-4651 mimics (synthesized by Shanghai Genechem Co.). After $48 \mathrm{~h}$ miR-4651 expression levels were determined by qPCR.

Ago2 immunoprecipitation (Ago2-IP). For each condition, six million NSCLC cells were trypsinized, homogenized (using the commercial lysis buffer from Beyotime, Wuxi, China), and cell lysates subjected to the Ago2-IP (with biotinylated miR-4651, generated by Shanghai Genechem Co.) under the previously 
described protocol [29]. As the negative control IP experiments were also carried out using the nonimmune IgG beads (Sigma). The IP-pull down RNA was used as template for qPCR assay, and results were quantified to input controls ("Input").

Cell-counting kit 8 (CCK-8) assay. NSCLC cells with the applied genetic treatments were trypsinized, counted, and inoculated into the 96-well tissue-culturing plates (at the initial density of 4,000 cells per well), incubated at $37^{\circ} \mathrm{C}$ for $72 \mathrm{~h}$. In each well $10 \mu \mathrm{L}$ of CCK-8 reagent (Dojindo, Kumamoto, Japan) was added and incubated for $2 \mathrm{~h}$, with CCK-8 absorbance examined at the wavelength of $450 \mathrm{~nm}$.

Colony formation. A549 cells with applied genetic treatments were initially seeded at 3,000 per well into the 12-well tissue-culturing plates. After seven days colonies were stained, with large colonies $(>100$ cells/per colony) counted manually.

Transwell migration assay. NSCLC cells with the applied genetic treatments were trypsinized and suspended into serum-free medium. For each treatment $5 \times 10^{4}$ cells were added to the upper surfaces of Matrigel-coated Transwell chambers (BD Biosciences, Shanghai, China) with $12 \mu \mathrm{m}$ pore-size. The lower chambers were filled with complete medium with $15 \%$ FBS. Cells were allowed to migrate for $24 \mathrm{~h}$. Cells in the upper chambers were removed, with the invasive cells in the lower chambers fixed (by $4 \%$ paraformaldehyde), stained and counted.

EdU assay. NSCLC cells with the indicated genetic modifications were trypsinized and initially seeded into the six-well tissue-culture plates (at $2 \times 10^{5}$ cells per well), and cultured for $48 \mathrm{~h}$. Using an EdU (5-ethynyl20-deoxyuridine) Apollo-567 Kit (RiboBio, Guangzhou, China), cell proliferation ratio (EdU/DAPI $\times 100 \%$ ) were determined. For each condition 500 nuclei in five random views were included.

Annexin V FACS assay of cell apoptosis. NSCLC cells with the indicated genetic modifications were trypsinized and stained with Annexin V-FITC and PI dyes $(25 \mu \mathrm{g} / \mathrm{mL}$ each) for $30 \mathrm{~min}$ at room temperature. Using a flow cytometry machine (Beckman Coulter, Brea, CA), the Annexin V positive staining cells were gated.

Western blotting. Total proteins of human tissue samples or cultured cells (with the applied genetic treatments) were isolated through a Total Protein Extraction kit (KeyGen Biotech Co., Nanjing, China), with the protein concentrations determined by the BCA assay kits (Sigma). The protein lysates ( $40 \mu \mathrm{g}$ in each lane) were separated by $10-12 \%$ SDS-PAGE gels, and transferred to polyvinylidene difluoride (PVDF) blots (EMD Millipore, Shanghai, China). Following blocking, the blots were incubated with the applied primary antibodies overnight at $4{ }^{\circ} \mathrm{C}$, washed and incubated with the corresponding secondary antibodies. The antibody-antigen binding was detected by the ECL Analysis system (GE Healthcare, Chicago, IL). Quantification of the blots using the ImageJ software was described early [30].

BRD4 knockout. A549 cells were initially seeded into the six-well plates $\left(1 \times 10^{5}\right.$ cells per well), transfected with the lenti-CRISPR/Cas9-BRD4-KO-GFP construct (provided by Dr. Zhao [31] at Soochow University) by Lipofectamine 2000. FACS-mediated sorting of the GFP-positive-A549 cells was performed 
to select monoclonal A549 cells, which were cultured in puromycin-containing complete medium. BRD4 knockout (KO) in the stable cells was screened by Western blotting and/or qPCR assays.

UTR mutant BRD4. The in vitro site-directed mutagenesis system (Genechem, Shanghai, China) was applied to generate 3'-UTR mutant BRD4 vector (at the miR-4651's binding site, 1716C >A), mut-BRD4. The construct was sub-cloned into the GV248 lentiviral vector, and added to A549 cells. Expression of the UTR mut-BRD4 was verified by Western blotting.

In vivo tumor growth assay. A549 cells with the applied genetic treatments were collected and injected subcutaneously into the right flanks of the severe combined immunodeficient mice (SCID) mice (all female, 18-19.5 grams in weights, purchased from Soochow University Animal facility, Suzhou, China). When the volume reached approximately $100 \mathrm{~mm}^{3}$ for each tumor ("Day-0"), the recordings were started. Tumor volumes were calculated through a previously-described protocol [32]. All procedures involving animals were approved by the Experimental Animal Ethical Committee of Soochow University, in accordance to the Declaration of Helsinki and the guidelines of the Ethics Committee of Soochow University.

Statistical analysis. Data were presented as the mean \pm standard deviations (SD), analyzed by SPSS 19.0 software (SPSS Co. Chicago, CA). The one-way ANOVA followed by Student-Newman-Keuls post hoc test was performed to determine statistical significance between multiple groups. When comparing difference between two groups the two-tailed Student's t-test (Excel 2007) was utilized. Values of $P<0.05$ were considered as statistically different.

\section{Results}

\section{1. miR-4651 targets BRD4 in human lung cancer cells}

First, the miRNA database, TargetScan (V7.2, http://www.targetscan.org), was consulted to explore possible BRD4-targeting miRNAs binding to its 3'-UTR. The possible miRNAs were further verified through other miRNA databases, including miRBase, miRNAmap and miRTarbase. The bioinformatics analyses identified one particular miRNA, miR-4651, potentially targets the 3'-UTR (at position 1715-1722) of BRD4 (Figure 1A). To study whether miR-4651 can affect BRD4 expression, a lentivirus-packaged pri-miR-4651 construct, LV-pri-miR-4651, was established and transduced to A549 NSCLC cells. With selection by puromycin two stable cell lines, LV-pri-miR-4651-sLine-1/2, were established. qPCR analyses showed that the mature miR-4651 levels increased over 11-12 folds in stable cells with LV-pri-miR-4651 (Figure 1B). Significantly, ectopic overexpression of miR-4651 led to a dramatic reduction of BRD4 3'-UTR activity (Figure 1C), suggesting that miR-4651 directly targets BRD4 in A549 cells. Further results demonstrated that LV-pri-miR-4651 led to downregulation of BRD4 mRNA (Figure 1D) and protein (Figure 1F). As a result, mRNA and protein expression of BRD4-dependent genes, including c-Myc, Bcl-2, cyclin D1 [6, 10, 
11, 33], was significantly downregulated (Figure $1 \mathrm{E}$ and F). These results imply that miR-4651 possibly targets BRD4 in A549 lung cancer cells.

To support that BRD4 is the direct target of miR-4651, two mutant miR-4651 mimics ("Mut1/2"), containing mutations within the binding sites of BRD4's 3-UTR (see Figure 1G), as well as the wild-type (WT) miR-4651 mimic, were transfected to A549 cells. As shown, only the WT miR-4651 mimic, but not the mutants, inhibited BRD4's 3-UTR activity (Figure 1H) and downregulated BRD4 mRNA levels (Figure 1I). We also tested whether miR-4651 can exert similar activity in primary cancer cells. The primary human lung cancer cells, provided by Dr. Jiang [26, 27], were derived from three written-informed consent NSCLC patients: Pri-Ca-1/2/3. As shown, LV-pri-miR-4651 led to significant increase of mature miR-4651 expression in the primary cancer cells (Figure $1 \mathrm{~J}$ ), causing potent BRD 4 mRNA downregulation (Figure $1 \mathrm{~K})$. Western blotting assay results, Figure $1 \mathrm{~L}$, demonstrated that protein expression of BRD4 and its targets, c-Myc, Bcl-2, cyclin D1, was decreased in Pri-Ca-1 cells with miR-4651 overexpression. Similar results were obtained in two other primary cancer cells (Data not shown). To further confirm that miR4651 directly targets BRD4 mRNA, the Ago2 immunoprecipitation (Ago2-IP) experiments were carried out. In A549 cells and primary human lung cancer cells (Pri-Ca-1/2/3), BRD4 mRNA was indeed enriched in the Ago2-IP (IP with miR-4651) in the tested NSCLC cells (Figure 1M). Collectively, these results show that miR-4651 is a BRD4-targeting miRNA in human lung cancer cells.

\section{2. miR-4651 overexpression inhibits lung cancer cell growth, proliferation and migration, while activating cell apoptosis}

We next tested whether ectopic overexpression of miR-4651 could affect the function of lung cancer cells. Results of cell counting assay, in Figure 2A, demonstrated that LV-pri-miR-4651-expressing A549 cells (see Figure 1) grew significantly slower than the parental control cells. Furthermore, miR-4651overexpressed stable cells presented with reduced cell viability (CCK-8 OD, Figure 2B). A549 cell colony formation was potently inhibited as well with miR-4651 overexpression (Figure 2C). Additionally, EdU incorporation in the two stable cell lines with LV-pri-miR-4651 was largely suppressed, as compared to that in the control cells (Figure 2D). Analyzing cell migration, by the "Transwell" assays, showed that miR4651 overexpression decreased the number of migrated A549 cells (Figure 2E). These results show that ectopic overexpression of miR-4651 inhibited A549 cell growth, proliferation and migration.

Testing the potential function of miR-4651 on cell apoptosis, we show that LV-pri-miR-4651 significantly increased the number of the Annexin V-positive A549 cells (Figure 2F), and induced cleavages of caspase-3 and poly (ADP) ribose polymerase (PARP) (Figure $\mathbf{2 G )}$ ). These results indicated the pro- 
apoptosis activity by miR-4651 overexpression. The non-sense control miRNA vector ("miRC") had no significant effect on the functions of A549 cells (Figure 2A-G). In the primary human lung cancer cells, PriCa-1/2/3, LV-pri-miR-4651-induced miR-4651 overexpression (see Figure 1) significantly inhibited CCK-8 viability (Figure $\mathbf{2 H}$ ), EdU incorporation (Figure $2 \mathrm{I}$ ) and cell migration (Figure $\mathbf{2} \mathrm{J}$ ). A significant apoptosis activation, evidenced by an increased Annexin V-staining, was detected in miR-4651-overexpressed primary cancer cells (Figure $\mathbf{2 K}$ ). Together, we show that miR-4651 overexpression inhibited lung cancer cell growth, proliferation and migration, while activating cell apoptosis.

\section{3. miR-4651 inhibition increases BRD4 expression and promotes lung cancer cell growth, proliferation and migration}

To inhibit miR-4651 expression, a lentivirus-packed pri-miR-4651 anti-sense sequence, LV-antagomiR4651, was transduced to A549 NSCLC cells. Followed by puromycin selection, two stable cell lines, sLine$1 /-2$, were established. As compared to control cells with control anti-sense sequence ("anta-C"), mature miR-4651 levels decreased over 90\% in LV-antagomiR-4651-expressing A549 cells (Figure 3A). On the contrary, LV-antagomiR-4651 increased BRD4 3'-UTR activity (Figure 3B) as well as BRD4 mRNA/protein levels (Figure $3 \mathrm{C}$ and $\mathrm{E}$ ). mRNA and protein expression of BRD4-dependent genes, c-Myc, Bcl-2 and cyclin D1, was upregulated as well in LV-antagomiR-4651-expressing A549 cells (Figure 3D and E). Therefore, miR-4651 inhibition increased BRD4 expression in A549 cells.

Functional studies show that in A549 cells LV-antagomiR-4651 increased CCK-8 OD (Figure 3F) and BrdU incorporation (Figure 3G), promoting cell survival and proliferation. Furthermore, A549 cell migration, tested by counting migrated cells in the "Transwell" assays, was significantly boosted with miR-4651 inhibition (Figure $3 \mathrm{H}$ ). Similarly in Pri-Ca-1/2/3 primary human NSCLC cells, LV-antagomiR-4651 induced 80-90\% reduction of miR-4651 expression (Figure 3I), but upregulated BRD4 mRNA (Figure $3 \mathrm{~J}$ ). Protein expression of BRD4 and its targets, c-Myc, Bcl-2, cyclin D1, was elevated (Figure 3K, in Pri-Ca-1 cells). LVantagomiR-4651-expressing primary cancer cells presented with increased cell growth (Figure 3L), proliferation (Figure $3 \mathrm{M}$ ) and migration (Figure $\mathbf{3 N}$ ), when compared to control cells with anta-C (Figure 3L-N). Together, the functional results show that miR-4651 inhibition promoted lung cancer cell growth, proliferation and migration.

\subsection{BRD4 is the main target of miR-4651 in lung cancer cells}


To test that BRD4 is the main target of miR-4651 in lung cancer cells, an 3'-UTR mutant (at miR-4651binding sites, 1716C>A) BRD4 lentiviral construct ("mut-BRD4") was established, which was transduced to LV-pri-miR-4651-expressing stable A549 cells. As shown the mut-BRD4 restored mature BRD4 mRNA (Figure 4A) and BRD4 protein (Figure 4B) expression in miR-4651-overexpressed cells (LV-pri-miR-4651sLine-1, Figure 4C). LV-pri-miR-4651-induced downregulation of BRD4-tareget genes, c-Myc, Bcl-2, cyclin D1, was reversed by the mut-BRD4 as well (Figure 4B). Importantly, the mutant BRD4 abolished LV-primiR-4651-induced inhibitions on A549 cell growth (cell counting results, Figure 4D), proliferation (EdU incorporation, Figure 4E) and migration (Figure 4F). These results show that restoring BRD4 expression, by the 3'-UTR mutant BRD4, reversed miR-4651 overexpression-induced inhibitions on A549 cells, suggesting that BRD4 is the main target of miR-4651 in A549 cells.

Based on the results, we further hypothesized that BRD4 depletion should mimic miR-4651 overexpression-induced actions in NSCLC cells. The CRISPR/Cas9-BRD4-KO construct (provided by Dr. Zhao [31] at Soochow University), containing the small guide RNA (sgRNA) against BRD4, was transduced to A549 NSCLC cells. Following GFP sorting and puromycin selection two stable cell lines, BRD4-KO-sLine-1/2, were established, showing depleted BRD4 mRNA (Figure $\mathbf{4 G )}$ ) and protein (Figure $\mathbf{4 H}$ ) expression. In the BRD4-KO A549 cells, c-Myc, Bcl-2, cyclin D1 protein expression was significantly downregulated (Figure 4H). BRD4 KO potently inhibited A549 cell proliferation (EdU incorporation, Figure 4I) and migration (Figure 4J), mimicking miR-4651 overexpression-induced activity. Importantly, in the BRD4-KO cells, miR-4651 overexpression (by LV-pri-miR-4651, see Figure 1) or inhibition (by LVantagomiR-4651, see Figure 3) was completely ineffective on cell proliferation (EdU incorporation, Figure $\mathbf{4 K}$ ) and migration (Figure 4L). Although both genetic treatments significantly altered miR-4651 expression (Figure 4M). These results show that miR-4651 was completely ineffective in BRD4-KO cells, further supporting that BRD4 is the primary target of miR-4651 in A549 cells.

\section{5. miR-4651 overexpression inhibits A549 cell growth in SCID mice}

To study the potential role of miR-4651 on NSCLC cell growth in vivo, LV-pri-miR-4651-expressing stable A549 cells (see Figure 1 and 2) and miRC-expressing control A549 cells were inoculated into the right flanks of the SCID mice. Testing tumor growth by recording tumor volumes, Figure $5 \mathrm{~A}$, demonstrated that miR-4651-overexpressed A549 xenografts grew significantly slower than control A549 xenografts expressing miRC. Calculating daily tumor growth, by the formula (tumor volume at Day-35-tumor volume at Day-0)/35, showed that A549 xenograft growth was significantly inhibited with miR-4651 overexpression (Figure 5B). The mice body weights between the two groups were not significantly different (Figure 5B), neither did we notice any signs of apparent toxicities. 
At Day-7 and Day-14, one tumor of each group (total four xenograft tumors) was individually isolated, and tissue lysates subjected to GPCR and Western blotting assays. As shown, the mature miR-4651 levels were significantly elevated in LV-pri-miR-4651-expressing A549 xenografts (about ten folds vs. control tumors, Figure 5D). Contrarily, BRD4 mRNA (Figure 5E) and protein (Figure 5F) levels were decreased. Expression of BRD4-dependent genes, $c-M y c, B c l-2$, cyclin D1, was also significantly downregulated in miR-4651-overexpressed A549 xenograft tissues (Figure 5E and F). These results together show that miR4651 overexpression inhibited BRD4 expression and A549 xenograft growth in SCID mice.

\section{6. miR-4651 is downregulated in human lung cancer tissues, correlating with upregulation of BRD4 and its pathway genes}

At last we tested expression of miR-4651 in human lung cancer tissues. A total of ten (10) pairs of NSCLC tissues ("Ca") and paracancer lung epithelial tissues ("N") tissues were obtained. Testing miR-4651 expression, by qPCR, demonstrated that miR-4651 levels were significantly downregulated the "Ca" tissues $(\boldsymbol{P}<0.05$ vs. the "N" tissues, Figure 6A). Contrarily, BRD4 mRNA levels in the "Ca" tissues were significantly higher than those in the " $N$ " tissues (Figure 6B). mRNA levels of BRD4-dependent genes, $C$ $M y c, B c l-2$, cyclin D1, were also significantly elevated in the "Ca" tissues. Western blotting analyzing tissue lysates from three representative patients, Patient-1/-3/-4, showed that BRD4 and its targets (cMyc, Bcl-2, cyclin D1) were upregulated in NSCLC tissues (Figure 6C). Quantitative analyses integrating all 10 pairs of tissues confirmed that upregulation of BRD4, C-Myc, Bcl-2 and cyclin D1 proteins in "Ca" tissues was significant $(\boldsymbol{P}<0.05 \mathrm{vs}$. the "N" tissues, Figure 6D). These results together show that miR4651 is downregulated in human NSCLC tissues, correlating with upregulation of BRD4 and its target genes.

\section{Discussion}

The physiological function of miR-4651 is still largely elusive. Recent studies reported a higher serum level of miR-4651 in certain aflatoxin B1-positive hepatocellular carcinoma (HCC) patients [34]. Tan et al., demonstrated that miR-4651 targeted SMG9 and repressed the expression levels of SMG9, thus inhibiting nonsense-mediated mRNA decay (NMD) activity [35]. Our results show that miR-4651 specifically and directly targets BRD4 in human lung cancer cells. The Ago-2 IP experiments confirmed that miR-4651 directly binds to BRD4 mRNA in A549 cells. Significantly, in A549 cells and primary human NSCLC cells ectopic overexpression of miR-4651, by LV-pri-miR-4651, inhibited BRD4's 3-UTR activity and its expression, both were however significantly elevated in NSCLC cells with miR-4651 inhibition (by LVantagomiR-4651). Expression of BRD4-regulated genes, c-Myc, Bcl-2, cyclin D1 [6, 10, 11, 33], was also 
regulated by miR-4651 in A549 and primary human NSCLC cells. Therefore, miR-4651 targets and negatively regulates BRD4 in human NSCLC cells.

Recent studies have demonstrated that BRD4 is overexpressed in human lung cancer tissues/cells, serves as a valuable and novel oncotarget for potential cancer therapy $[7,8]$. The BRD4 pharmacological inhibitors (JQ1 and I-BET151) or BRD4 silencing (by targeted siRNAs) potently inhibited lung cancer cell progression in vitro and in vivo $[7,8]$. Here we show that miR-4651-induced silencing of BRD4 suppressed NSCLC cell progression. In vitro, forced overexpression of miR-4651, by LV-pri-miR-4651, inhibited NSCLC cell growth, proliferation and migration, while inducing apoptosis activation. In vivo, miR-4651overexpressed A549 xenografts grew significantly slower than control A549 xenografts in SCID mice. Contrarily, LV-antagomiR-4651-mediated miR-4651 inhibition further promoted NSCLC cell growth, proliferation and migration. Therefore, miR-4651-mediated BRD4 silencing could be a novel strategy to inhibit NSCLC cell progression.

MiR-4651 could have other potential targets, as suggested by studies in other human cells [34, 35]. The results of this study show that BRD4 should be the main target of miR-4651 in NSCLC cells. BRD4 reexpression, by an 3'-UTR mutant BRD4, reversed miR-4651 overexpression-induced inhibitions on A549 cells. Furthermore, BRD4 KO, using CRISPR/Cas9 strategy, mimicked miR-4651 overexpression-induced activity in A549 cells. More importantly, miR-4651 overexpression or inhibition was completely ineffective in the BRD4-KO A549 cells. Therefore, we conclude that miR-4651 inhibits NSCLC cell progression by silencing BRD4.

\section{Conclusion}

The efficiency of the current clinical NSCLC treatment options, including the traditional cancer resection, platinum-based chemotherapy and radiation as well as the latest targeted therapies, is far from satisfactory $[2,36]$. The results of this study show that miR-4651, being downregulated in human NSCLC tissues, could significantly inhibit proliferation of NSCLC cells by negatively regulating BRD4. It should represent as a novel therapy strategy for NSCLC.

\section{Abbreviations}

Ago2 immunoprecipitation (Ago2-IP); bromodomain-containing protein 4 (BRD4); epithelial-mesenchymal transition (EMT); fetal bovine serum (FBS); knockout (KO); microRNAs (miRNAs); microRNA-4651 (miR4651); noncoding; RNAs (ncRNAs)non-small cell lung cancer (NSCLC); positives transcription elongation factor b (P-TEFb); quantitative real-time PCR (qPCR); standard deviations (SD).

\section{Declarations}

Ethical Approval and Consent to participate. This study was approved by the Ethics Committee of Soochow University. 
Consent for publication. Not applicable.

Availability of data and material. All data generated during this study are included in this published article. Data will be made available upon request.

Competing interests. The authors declare that they have no competing interests.

Funding. This project was supported by Natural Science Foundation of China (81773192 and 81902715$)$. The funders had no role in the study design, data collection and analysis, decision to publish, or preparation of the manuscript.

Authors' contributions. All listed authors designed the study, performed the experiments and the statistical analysis, and wrote the manuscript. All authors have read the manuscript and approved the final version.

Acknowledgements. We thank Dr. Li from Wenzhou Medical University for the manuscript editing and study design.

Authors' information. Not applicable.

\section{References}

1. Siegel RL, Miller KD, Jemal A: Cancer statistics, 2018. CA Cancer J Clin 2018, 68:7-30.

2. Rosell R, Karachaliou N: Lung cancer in 2014: optimizing lung cancer treatment approaches. Nat Rev Clin Oncol 2015, 12:75-76.

3. Vestergaard HH, Christensen MR, Lassen UN: A systematic review of targeted agents for non-small cell lung cancer. Acta Oncol 2018, 57:176-186.

4. Yang L, Wang L, Zhang Y: Immunotherapy for lung cancer: advances and prospects. Am J Clin Exp Immunol 2016, 5:1-20.

5. Horn L, Reck M, Spigel DR: The Future of Immunotherapy in the Treatment of Small Cell Lung Cancer. Oncologist 2016, 21:910-921.

6. Devaiah BN, Singer DS: Two faces of brd4: mitotic bookmark and transcriptional lynchpin. Transcription 2013, 4:13-17.

7. Gao Z, Yuan T, Zhou X, Ni P, Sun G, Li P, Cheng Z, Wang X: Targeting BRD4 proteins suppresses the growth of NSCLC through downregulation of elF4E expression. Cancer Biol Ther 2018, 19:407-415.

8. Liao YF, Wu YB, Long X, Zhu SQ, Jin C, Xu JJ, Ding JY: High level of BRD4 promotes non-small cell lung cancer progression. Oncotarget 2016, 7:9491-9500.

9. Hajmirza A, Emadali A, Gauthier A, Casasnovas O, Gressin R, Callanan MB: BET Family Protein BRD4: An Emerging Actor in NFkappaB Signaling in Inflammation and Cancer. Biomedicines 2018, 6.

10. Fu LL, Tian M, Li X, Li JJ, Huang J, Ouyang L, Zhang Y, Liu B: Inhibition of BET bromodomains as a therapeutic strategy for cancer drug discovery. Oncotarget 2015, 6:5501-5516. 
11. Shi J, Vakoc CR: The mechanisms behind the therapeutic activity of BET bromodomain inhibition. Mol Cell 2014, 54:728-736.

12. Treiber T, Treiber N, Meister G: Regulation of microRNA biogenesis and its crosstalk with other cellular pathways. Nat Rev Mol Cell Biol 2019, 20:5-20.

13. Jonas $\mathrm{S}$, Izaurralde $\mathrm{E}$ : Towards a molecular understanding of microRNA-mediated gene silencing. Nat Rev Genet 2015, 16:421-433.

14. Pasquinelli $A E$ : MicroRNAs and their targets: recognition, regulation and an emerging reciprocal relationship. Nat Rev Genet 2012, 13:271-282.

15. Calin GA, Croce CM: MicroRNA signatures in human cancers. Nat Rev Cancer 2006, 6:857-866.

16. Uddin A, Chakraborty S: Role of miRNAs in lung cancer. J Cell Physiol 2018.

17. Hao NB, He YF, Li XQ, Wang K, Wang RL: The role of miRNA and IncRNA in gastric cancer. Oncotarget 2017, 8:81572-81582.

18. Iqbal MA, Arora S, Prakasam G, Calin GA, Syed MA: MicroRNA in lung cancer: role, mechanisms, pathways and therapeutic relevance. Mol Aspects Med 2018.

19. Ulivi P, Foschi G, Mengozzi M, Scarpi E, Silvestrini R, Amadori D, Zoli W: Peripheral blood miR-328 expression as a potential biomarker for the early diagnosis of NSCLC. Int J Mol Sci 2013, 14:1033210342.

20. Wang ZX, Bian HB, Wang JR, Cheng ZX, Wang KM, De W: Prognostic significance of serum miRNA21 expression in human non-small cell lung cancer. J Surg Oncol 2011, 104:847-851.

21. Cheng Z, Ma R, Tan W, Zhang L: MiR-152 suppresses the proliferation and invasion of NSCLC cells by inhibiting FGF2. Exp Mol Med 2014, 46:e112.

22. Su Y, Wang Y, Zhou H, Lei L, Xu L: MicroRNA-152 targets ADAM17 to suppress NSCLC progression. FEBS Lett 2014, 588:1983-1988.

23. Xu W, Sun D, Wang Y, Zheng X, Li Y, Xia Y, Teng Y: Inhibitory effect of microRNA-608 on lung cancer cell proliferation, migration, and invasion by targeting BRD4 through the JAK2/STAT3 pathway. Bosn J Basic Med Sci 2019.

24. Guan H, You Z, Wang C, Fang F, Peng R, Mao L, Xu B, Chen M: MicroRNA-200a suppresses prostate cancer progression through BRD4/AR signaling pathway. Cancer Med 2019, 8:1474-1485.

25. Li D, Cui C, Chen J, Hu Z, Wang Y, Hu D: Long noncoding RNA UCA1 promotes papillary thyroid cancer cell proliferation via miR204mediated BRD4 activation. Mol Med Rep 2018, 18:3059-3067.

26. Yu H, Chen Y, Jiang P: Circular RNA HIPK3 exerts oncogenic properties through suppression of miR124 in lung cancer. Biochem Biophys Res Commun 2018, 506:455-462.

27. Zhang B, Lu HY, Xia YH, Jiang AG, Lv YX: Long non-coding RNA EPIC1 promotes human lung cancer cell growth. Biochem Biophys Res Commun 2018, 503:1342-1348.

28. Kang X, Kong F, Wu S, Liu Q, Yang C, Wu X, Zhang W: microRNA-612 suppresses the malignant development of non-small-cell lung cancer by directly targeting bromodomain-containing protein 4 . Onco Targets Ther 2019, 12:4167-4179. 
29. Hu HY, He L, Fominykh K, Yan Z, Guo S, Zhang X, Taylor MS, Tang L, Li J, Liu J, et al: Evolution of the human-specific microRNA miR-941. Nat Commun 2012, 3:1145.

30. Marshall J, Zhou XZ, Chen G, Yang SQ, Li Y, Wang Y, Zhang ZQ, Jiang Q, Birnbaumer L, Cao C: Antidepression action of BDNF requires and is mimicked by Galphai1/3 expression in the hippocampus. Proc Natl Acad Sci U S A 2018, 115:E3549-E3558.

31. Xiang T, Bai JY, She C, Yu DJ, Zhou XZ, Zhao TL: Bromodomain protein BRD4 promotes cell proliferation in skin squamous cell carcinoma. Cell Signal 2018, 42:106-113.

32. Pan SJ, Ren J, Jiang H, Liu W, Hu LY, Pan YX, Sun B, Sun QF, Bian LG: MAGEA6 promotes human glioma cell survival via targeting AMPKalpha1. Cancer Lett 2018, 412:21-29.

33. Chen R, Yik JH, Lew QJ, Chao SH: Brd4 and HEXIM1: multiple roles in P-TEFb regulation and cancer. Biomed Res Int 2014, 2014:232870.

34. Wu XM, Xi ZF, Liao P, Huang HD, Huang XY, Wang C, Ma Y, Xia Q, Yao JG, Long XD: Diagnostic and prognostic potential of serum microRNA-4651 for patients with hepatocellular carcinoma related to aflatoxin B1. Oncotarget 2017, 8:81235-81249.

35. Tan Y, Ma Z, Jin Y, Zong R, Wu J, Ren Z: MicroRNA 4651 regulates nonsense-mediated mRNA decay by targeting SMG9 mRNA. Gene 2019, 701:65-71.

36. Keith RL, Miller YE: Lung cancer chemoprevention: current status and future prospects. Nat Rev Clin Oncol 2013, 10:334-343.

\section{Table}

Table-1 Primers for qPCR assay utilized in this study

\begin{tabular}{|c|c|c|}
\hline Gene name & Forward primer $\left(5^{\prime}-3^{\prime}\right)$ & Reverse primer $\left(5^{\prime}-3^{\prime}\right)$ \\
\hline$B R D 4$ & CGCTATGTCACCTCCTGTTTGC & ACTCTGAGGACGAGAAGCCCTT \\
\hline$G A P D H$ & GTCTCCTCTGACTTCAACAGCG & ACCACCCTGTTGCTGTAGCCAA \\
\hline$M Y C$ & TCGGTCCTCGGATTCTCTGCTCT & GCCTCCAGCAGAAGGTGATCCA \\
\hline Cyclin D1 & TCTACACCGACAACTCCATCCG & TCTGGCATTTTGGAGAGGAAGTG \\
\hline$B c l-2$ & CATGTGTGTGGAGAGCGTCAA & GCCGGTTCAGGTACTCAGTCA \\
\hline $\operatorname{miR}-4651$ & CGGGGTGGGTGAGGTCGG & GAACATGTCTGCGTATCTC \\
\hline U6 & CTCGCTTCGGCAGCACAT & TTTGCGTGTCATCCTTGCG \\
\hline
\end{tabular}

\section{Figures}


Figure 1

A.

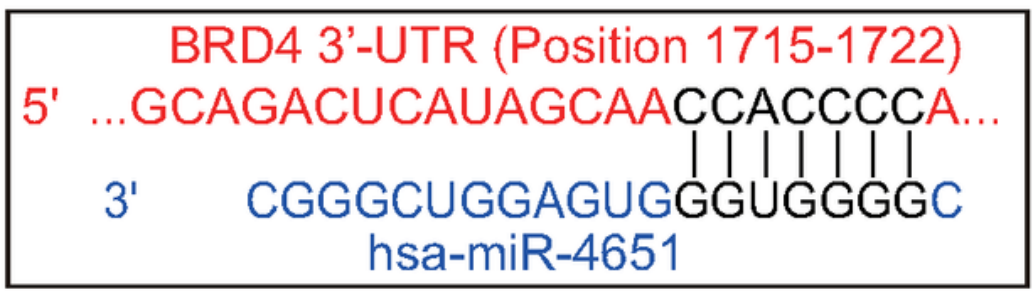

B.

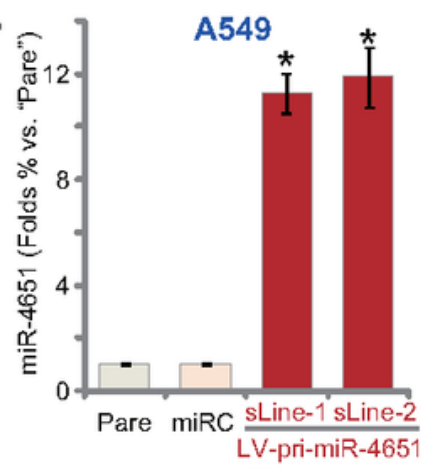

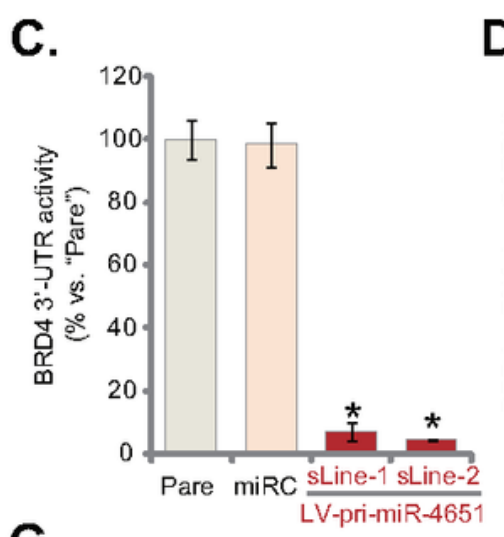

G.

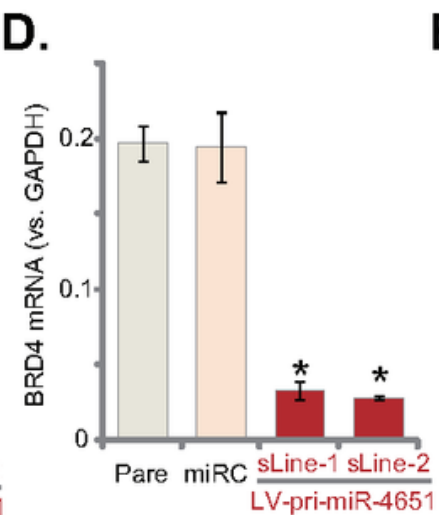

E.

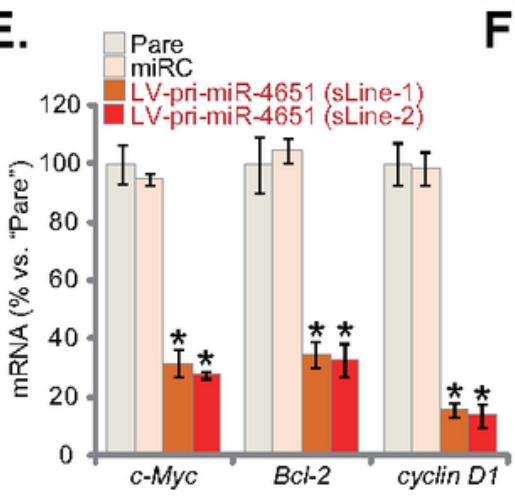

F.

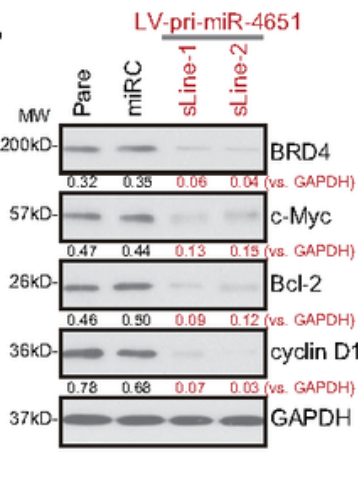

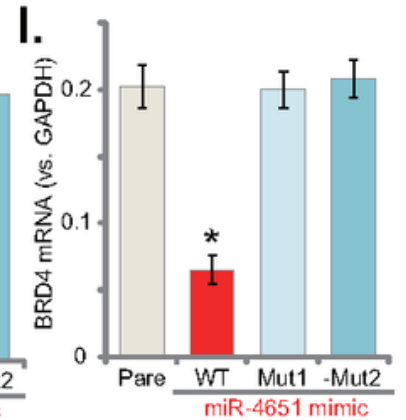

J.

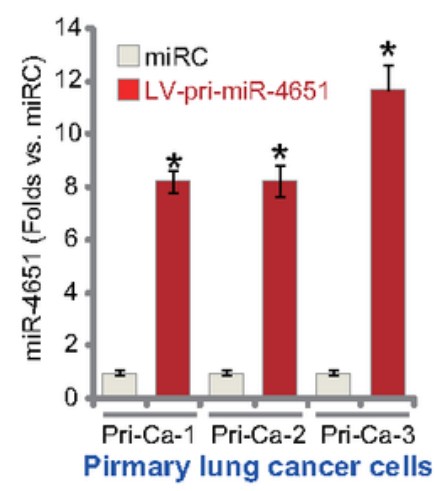

K.

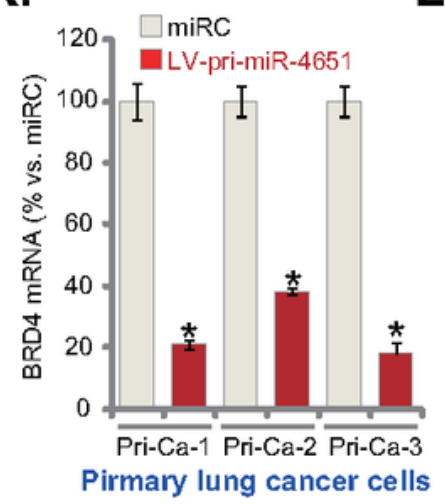

L.

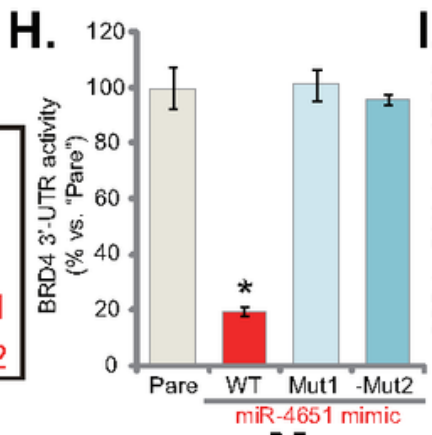

M.
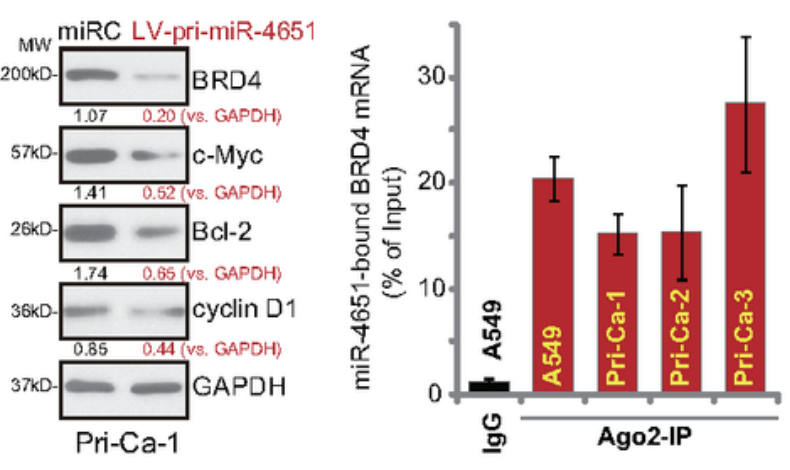

Figure 1

miR-4651 targets BRD4 in human lung cancer cells. miR-4651 putatively targets the BRD4 3'-UTR (untranslated region) at position 1715-1722 (A). A549 NSCLC cells were transduced with the lentiviral construct encoding pri-miR-4651 ("LV-pri-miR-4651") or the non-sense control miRNA vector ("miRC"), selected by puromycin to establish stable cells; The parental cells ("Pare") were utilized as the control cells; Expression of listed genes was tested by qPCR (B, D and E) and Western blotting (F) assays; The 
relative BRD4 3'-UTR activity was tested, with results normalized (C). A549 cells were transfected with wild-type (WT) or the mutant miR-4651 mimic (sequences listed in G, $500 \mathrm{nM}$, 48h), the relative BRD4 3'UTR activity $(\mathrm{H})$ and BRD4 mRNA expression (I, qPCR) were tested. The primary human lung cancer cells, Pri-Ca-1/2/3, were transduced with LV-pri-miR-4651 or miRC, and selected by puromycin to establish stable cells; Expression of mature miR-4651 (J), BRD4 mRNA (K) and listed proteins ( $\mathrm{L}$, in Pri-Ca-1 cells) was tested by qPCR and Western blotting assays. Ago2-IP experiments confirmed BRD4 mRNA enriched in Ago2-IP (IP with biotinylated miR-4651) in A549 and primary human lung cancer cells (M). Expression of listed proteins was quantified, normalized to the loading control GAPDH ( $F$ and $L$ ). Error bars stand for mean \pm standard deviation (SD, $n=5)$. * $P<0.05$ vs. "Pare"/"miRC" cells. Experiments in this figure were repeated four times, and similar results were obtained. 


\section{Figure 2}
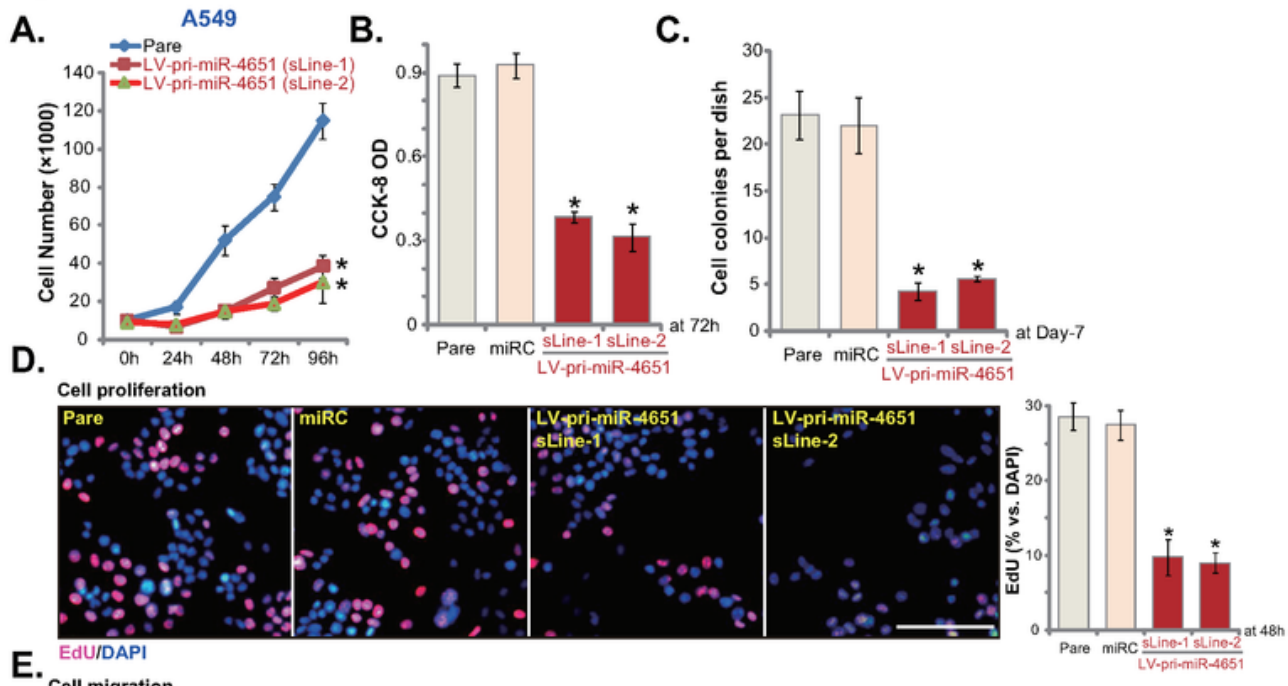

E. EdU/DAPI

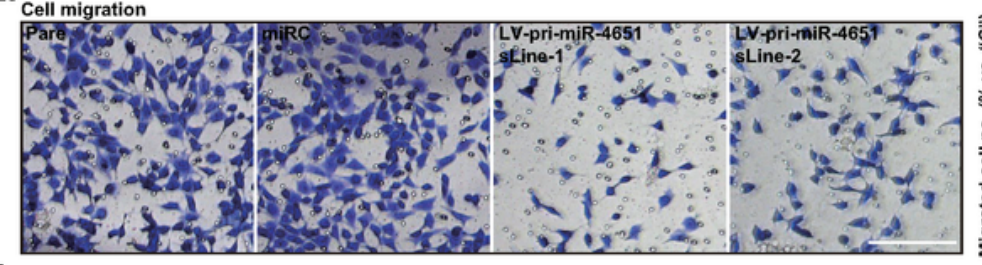

F. Pare

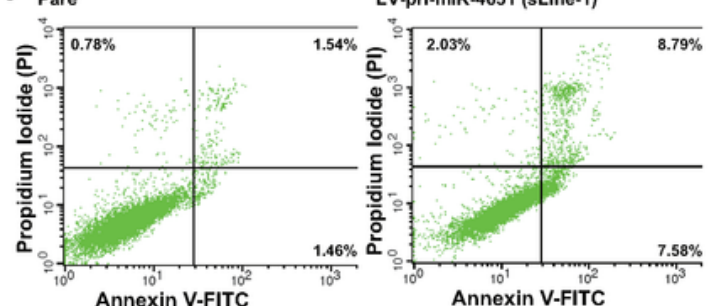

H.

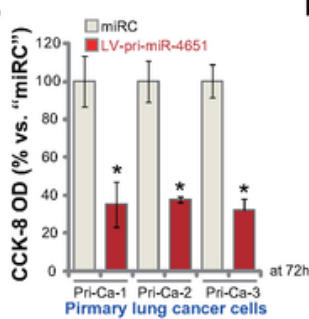

J.
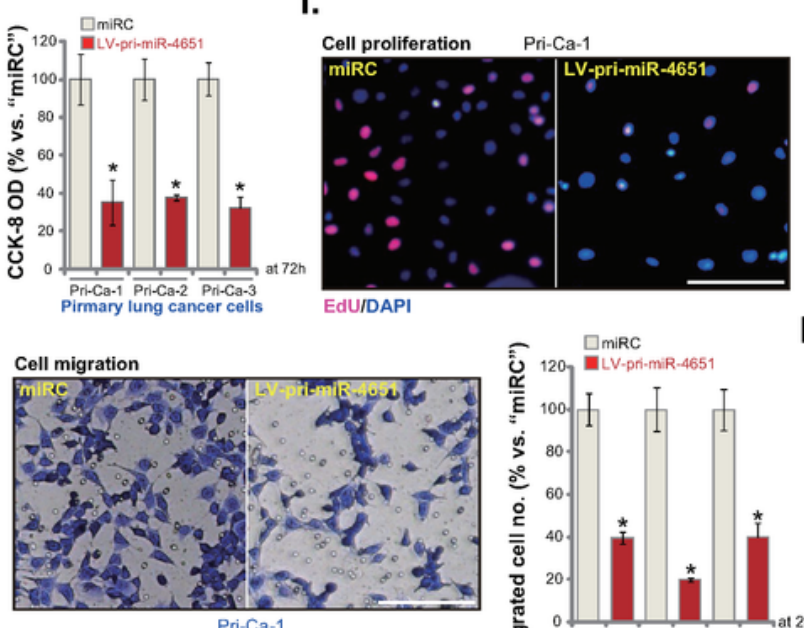

I.

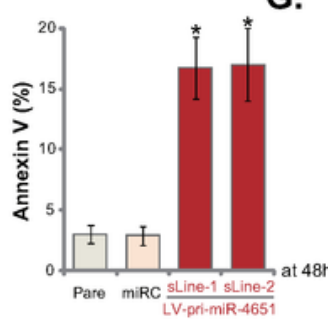

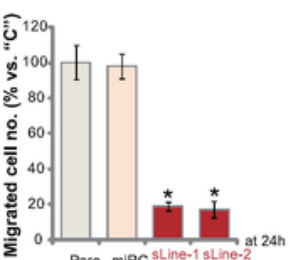

G.
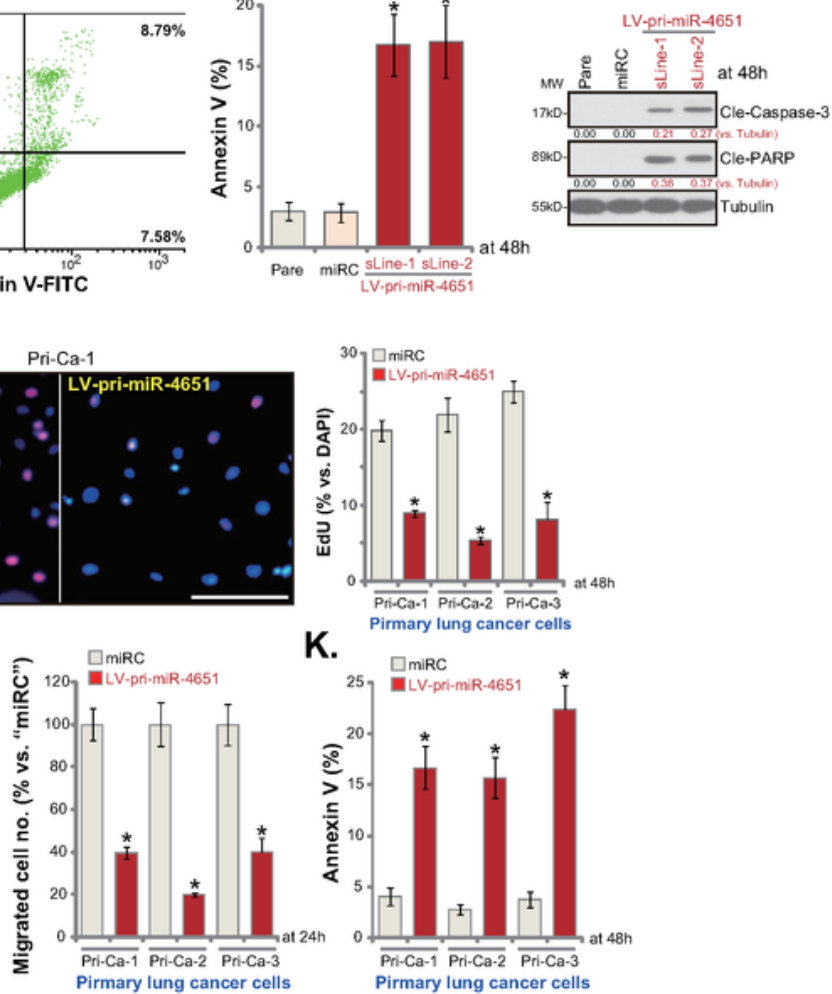

Figure 2

miR-4651 overexpression inhibits lung cancer cell growth, proliferation and migration, while activating cell apoptosis. The stable A549 cells with the lentivirus-packaged pri-miR-4651 construct ("LV-pri-miR4651", two lines) or the non-sense control miRNA vector ("miRC"), as well as the parental cells ("Pare") were cultured for applied time periods, cell growth $(A)$, viability (B), proliferation (C and D) and migration (E) were tested by the listed assays; Cell apoptosis was tested by Annexin V-FACS assay (F) and 
cleavages of caspase-3-PARP (G). The stable primary human lung cancer cells, Pri-Ca-1/2/3, with LV-primiR-4651 or miRC, were cultured for applied time periods, cell viability (CCK-8 assay, $\mathrm{H}$ ), proliferation (EdU incorporation, I), migration ("Transwell” assay, J) and apoptosis (Annexin V-FACS, K) were tested. Expression of listed proteins was quantified, normalized to the loading control Tubulin (G). Error bars stand for mean \pm standard deviation ( $S D, n=5)$. * $P<0.05$ vs. "Pare" /"miRC" cells. Experiments in this figure were repeated four times, and similar results were obtained. Bar=100 $\mu \mathrm{m}(\mathrm{D}, \mathrm{E}, \mathrm{I}$ and $\mathrm{J})$.

\section{Figure 3}

A.

$\mathbf{F}$.
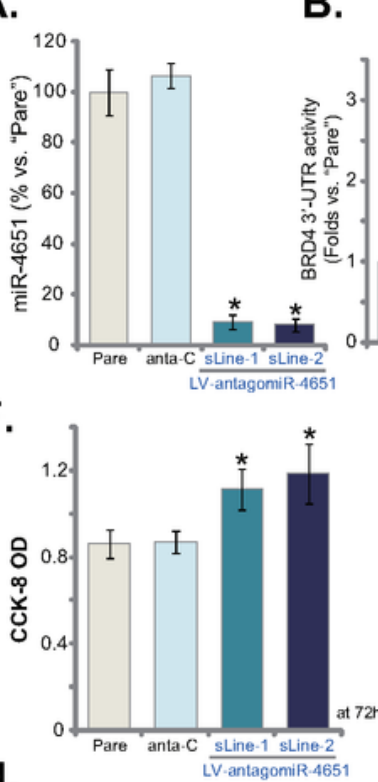

B.

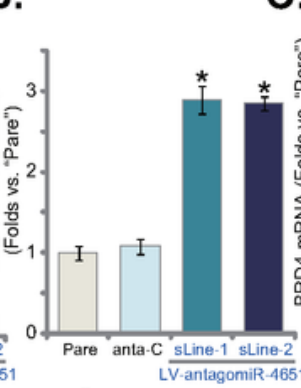

G.

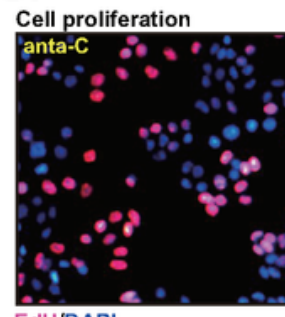

C.

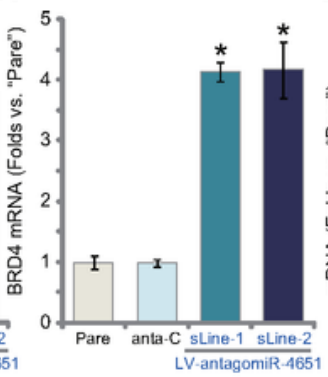

D.

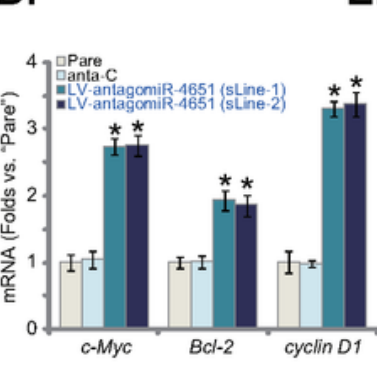

E.

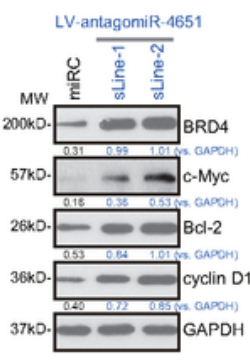

H.
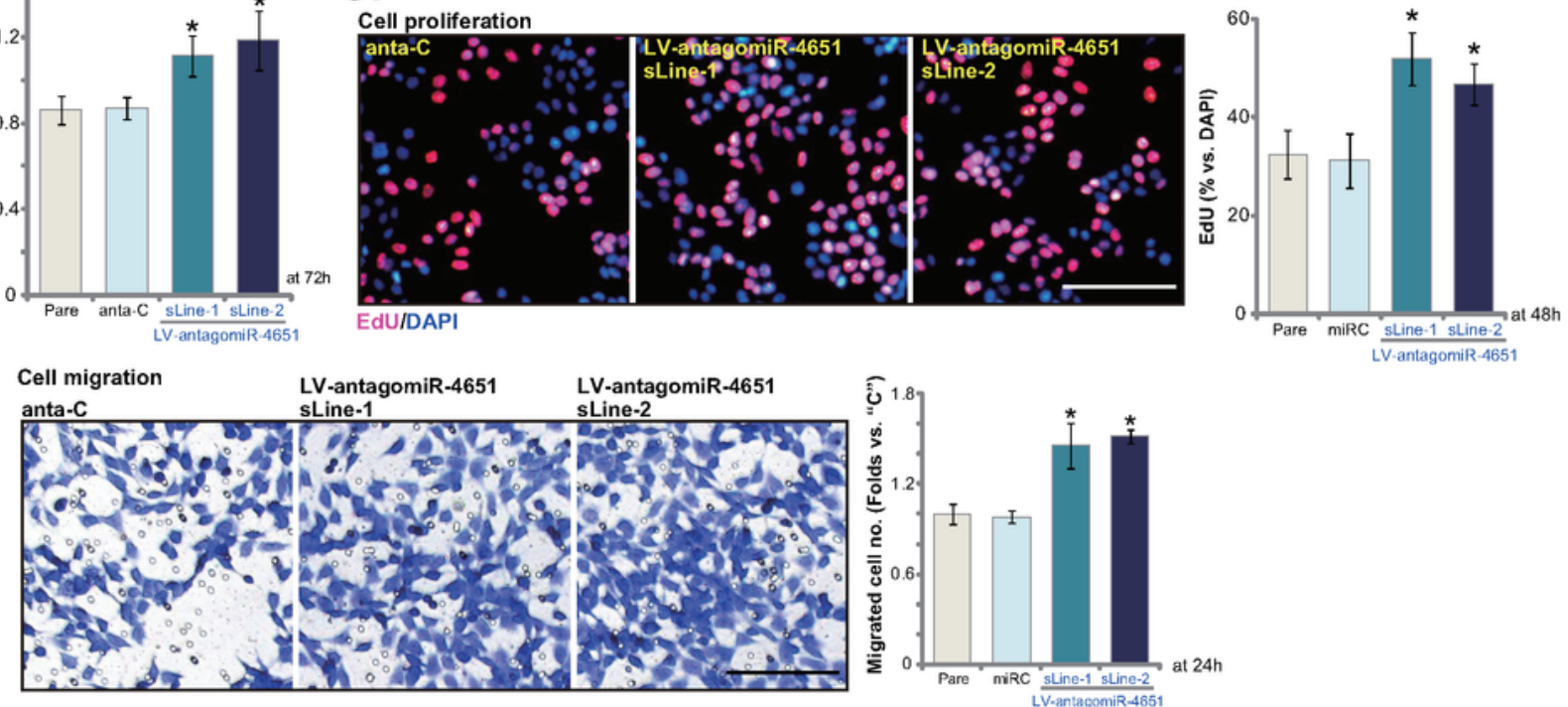

I.

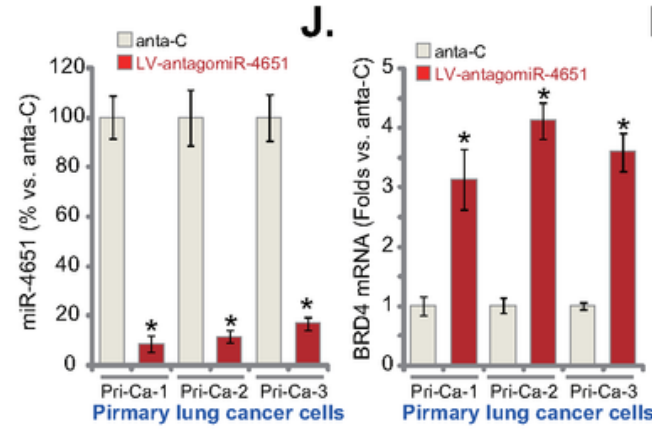

M.

K.

L. Danta-c

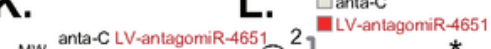
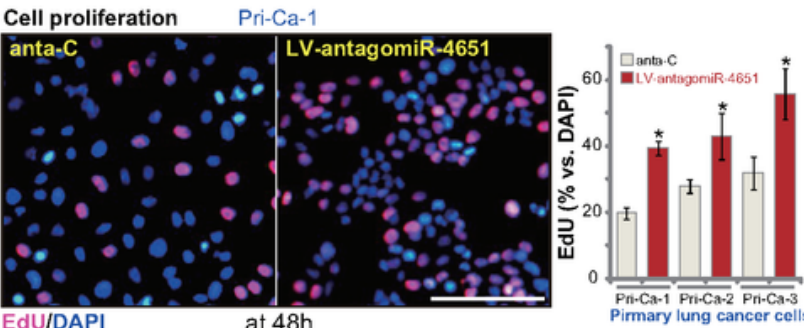

N.

Cell migration
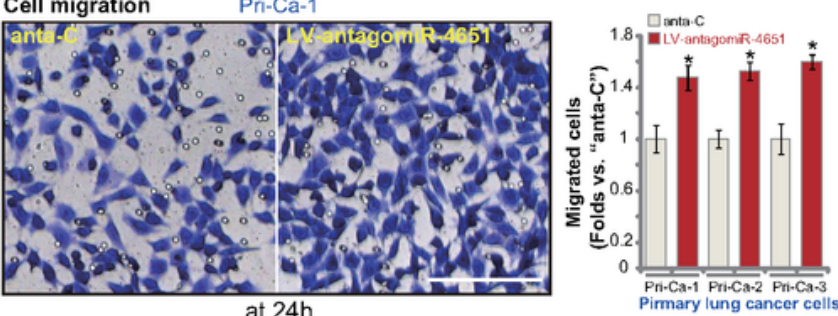

Figure 3 
miR-4651 inhibition increases BRD4 expression and promotes lung cancer cell growth, proliferation and migration. Stable A549 cells with the lentiviral construct encoding pri-miR-4651 anti-sense sequence (LVantagomiR-4651, two lines, sLine-1/2) or the control anti-sense sequence ("anta-C"), as well as the parental cells ("Pare") were cultured for applied time periods, expression of listed genes was tested (A, C, D and E), with relative BRD4 3'-UTR activity shown (B). Cells were further cultured for applied time periods, cell viability ( $F, C C K-8$ assay), proliferation ( $G$, EdU incorporation assay) and migration $(H$, "Transwell" assay) were tested. The stable primary human lung cancer cells, Pri-Ca-1/2/3, with LVantagomiR-4651 or anta-C, were established, relative expression of miR-4651 (I), BRD4 mRNA (J) and listed proteins (K) was tested by qPCR and Western blotting assays; Cells were further cultured for applied time periods, cell growth $(L)$, proliferation $(M)$ and migration $(N)$ were tested. Expression of listed proteins was quantified, normalized to the loading control GAPDH (E and K). Error bars stand for mean \pm standard deviation (SD, $n=5)$. * $P<0.05$ vs. "Pare"/"anta-C" cells. Experiments in this figure were repeated four times, and similar results were obtained. Bar=100 $\mu \mathrm{m}(\mathrm{G}, \mathrm{H}, \mathrm{M}$ and $\mathrm{N})$.

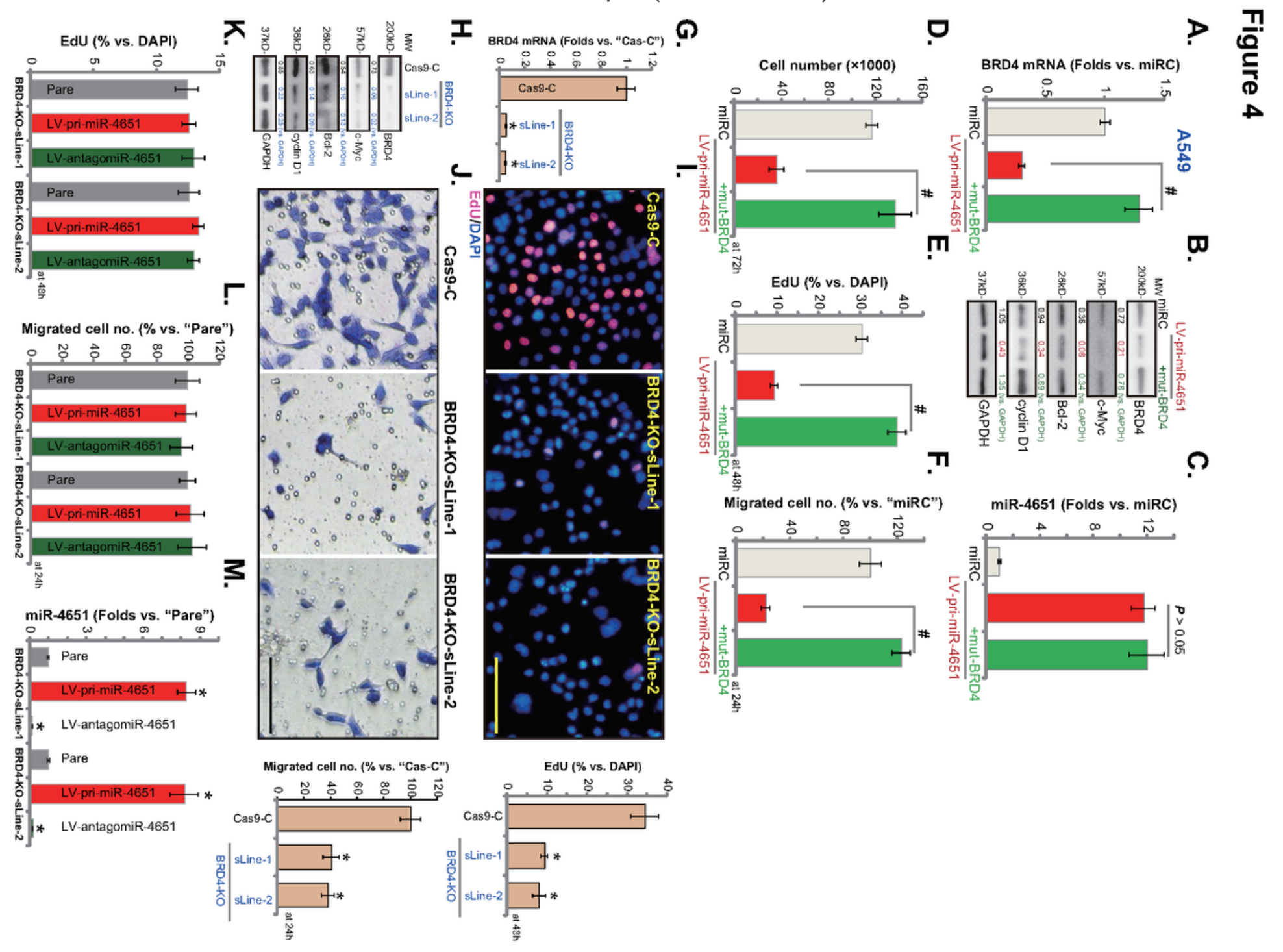

Figure 4 
BRD4 is the main target of miR-4651 in lung cancer cells. Stable A549 cells with the lentivirus-packaged pri-miR-4651 construct ("LV-pri-miR-4651") were further transduced with or without the 3'-UTR mutant BRD4 lentiviral construct ("+mut-BRD4"); Control cells were transduced with the non-sense control miRNA vector ("miRC"); Expression of listed genes in these cells was tested qPCR ( $A$ and $C$ ) and Western blotting (B) assays; Cells were further cultured for applied time periods, cell growth (cell counting assay, D), proliferation (EdU incorporation, E) and migration ("Transwell" assay, F) were tested. Stable A549 cells with the CRISPR/Cas9-BRD4-KO construct, BRD4-KO-sLine-1/2, as well as the control cells with the CRISPR/Cas9 non-sense sgRNA construct ("Cas9-C") were established; Expression of listed genes was tested by qPCR $(\mathrm{G})$ and Western blotting $(\mathrm{H})$ assays; Cell proliferation (EdU incorporation, I) and migration ("Transwell" assay, J) were tested. The BRD4-KO-sLine-1/2 were further infected with the lentiviruspackaged pri-miR-4651 construct ("LV-pri-miR-4651") or the lentivirus-packaged pri-miR-4651 anti-sense sequence (LV-antagomiR-4651), and cultured for applied time periods, cell proliferation (K) and migration $(\mathrm{L})$ were tested, with miR-4651 expression tested by qPCR (M). Expression of listed proteins was quantified, normalized to the loading control GAPDH (B and $\mathrm{H})$. Error bars stand for mean \pm standard deviation ( $S D, n=5)$. \# $P<0.05$ (A, D-F). * $P<0.05$ vs. "Cas9-C"/“Pare" cells. Experiments in this figure were repeated four times, and similar results were obtained. Bar=100 $\mu \mathrm{m}(\mathrm{I}$ and $\mathrm{J})$. 
Figure 5

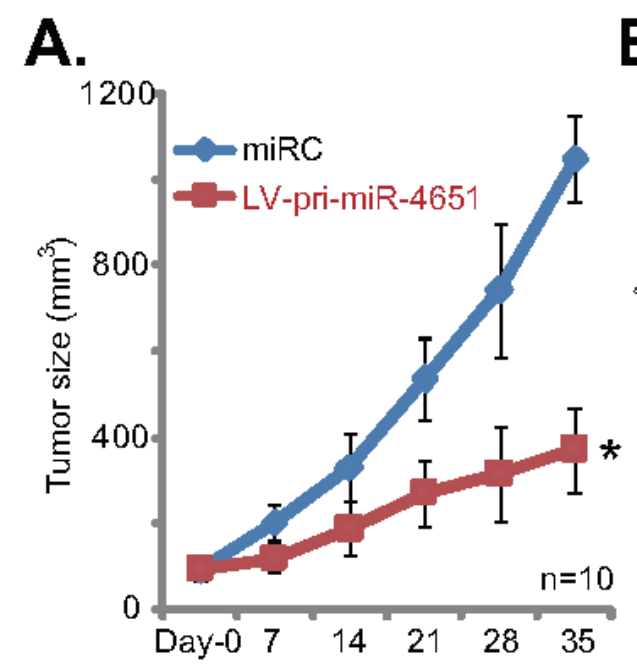

B. C.

D.
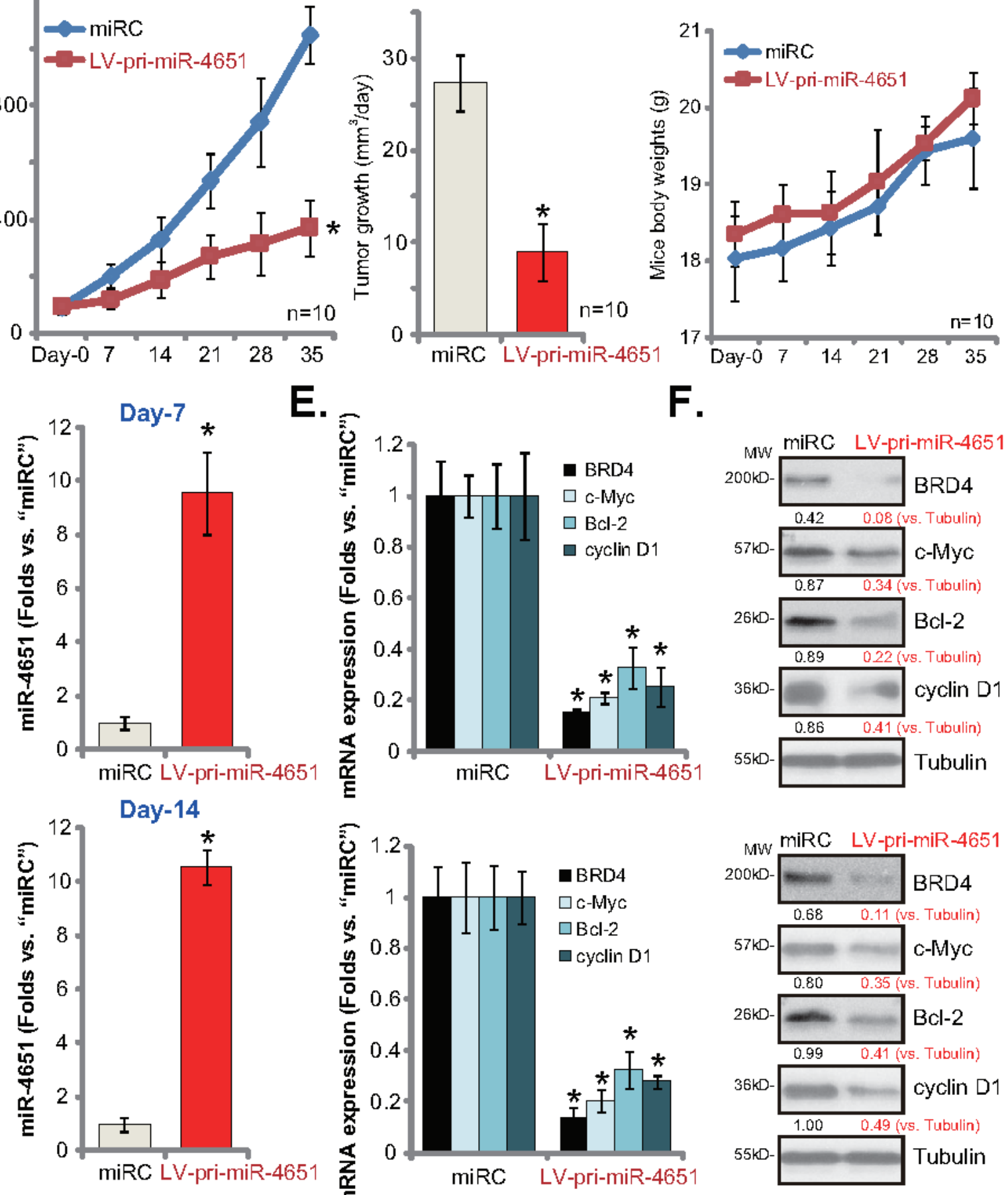

F.
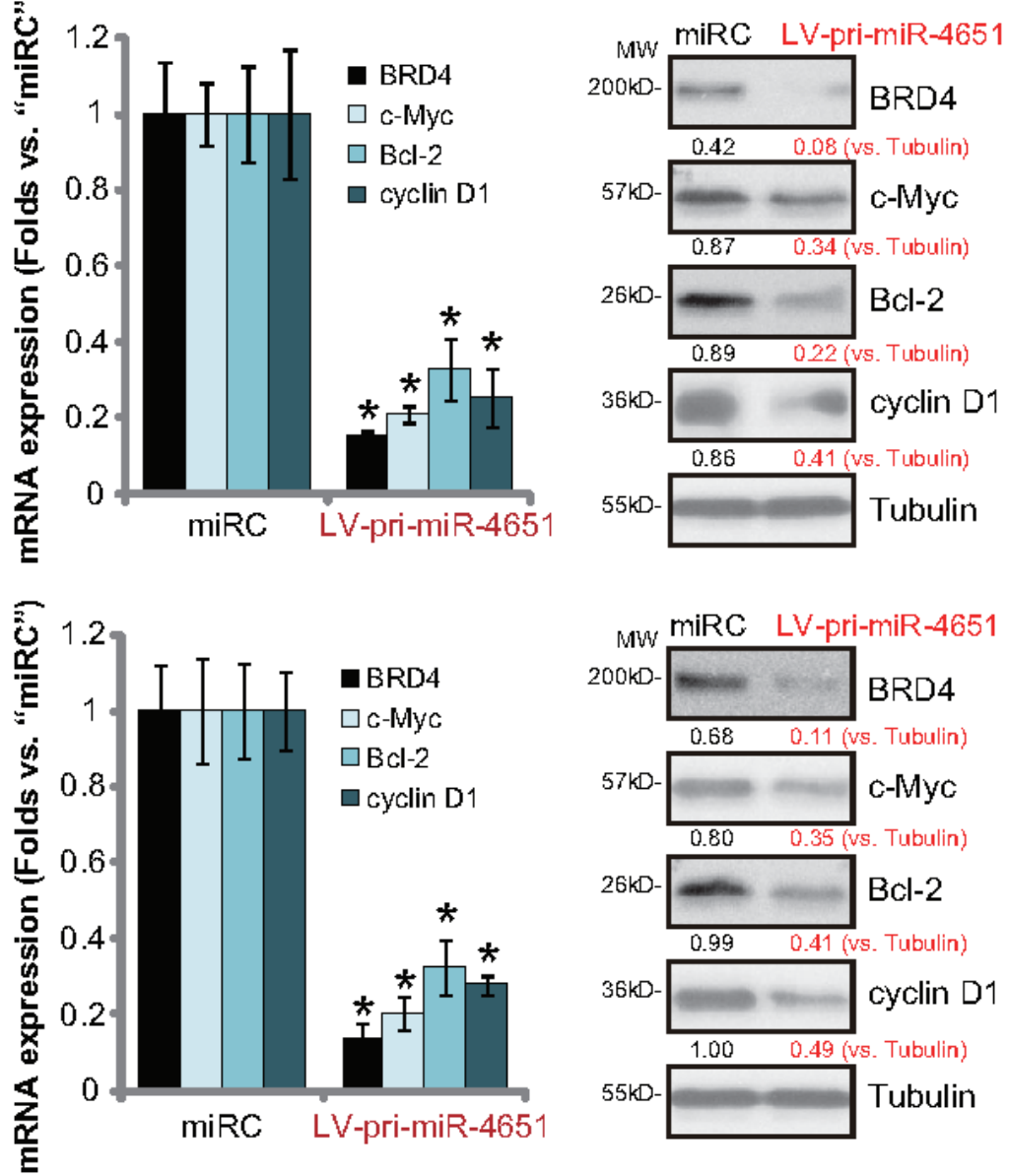

\section{Figure 5}

miR-4651 overexpression inhibits A549 cell growth in SCID mice. Stable A549 cells, with the lentiviruspackaged pri-miR-4651 construct ("LV-pri-miR-4651") or the non-sense control miRNA vector ("miRC"), were inoculated s.c. to SCID mice (10 mice per group, $n=10$ ); Within three weeks tumor xenografts were established. Afterwards, tumor volumes (A) and mice body weights (C) were recorded every seven days for a total of 35 days; Estimated average daily tumor growth was calculated (B); At Day-7 and Day-14, 
one A549 tumor xenograft per group was isolated (total four tumors), each tumor was randomly cut into five small pieces ( $n=5$, for $D-F)$, with expression of the listed genes in tumor lysates tested by qPCR ( $D$ and $E$ ) and Western blotting ( $F$ ) assays. Expression of listed proteins was quantified, normalized to the loading control Tubulin (F). * $p<0.05$ vs. "miRC" control tumors.

\section{Figure 6}
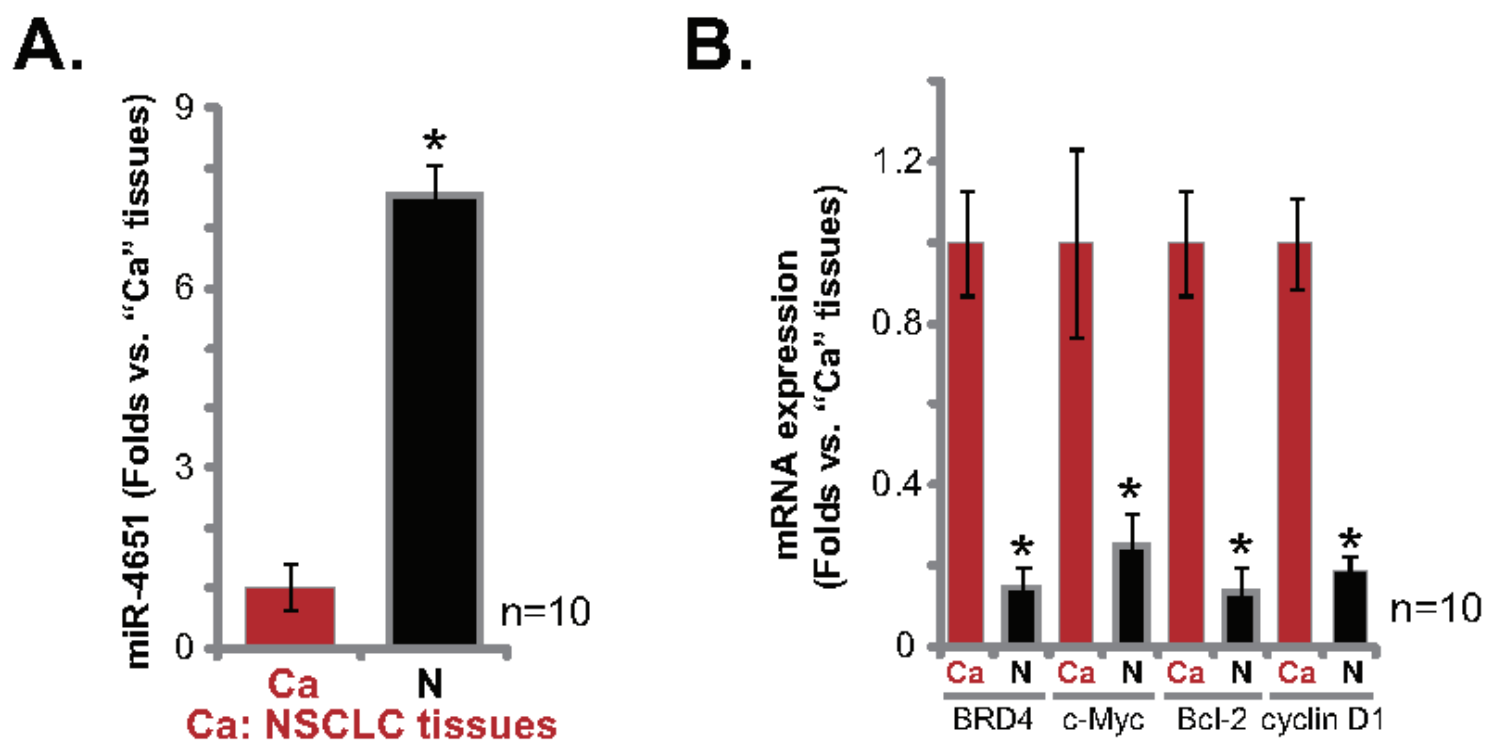

Ca: NSCLC tissues

$\mathrm{N}$ : Paracancer lung epithelial tissues

C.

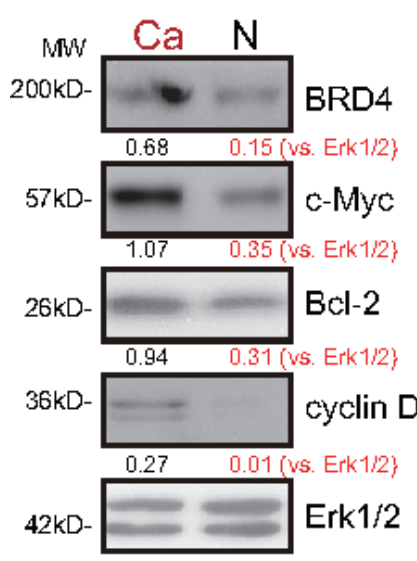

Patient-1

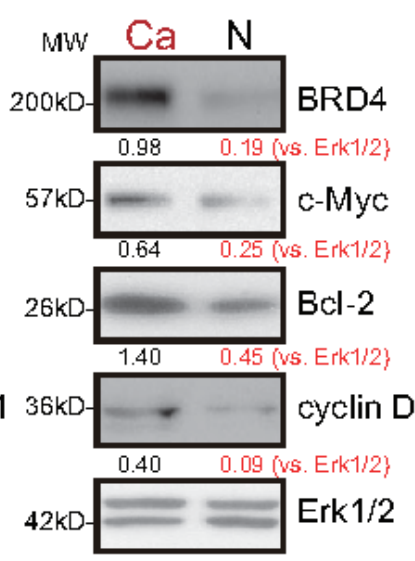

Patient-3

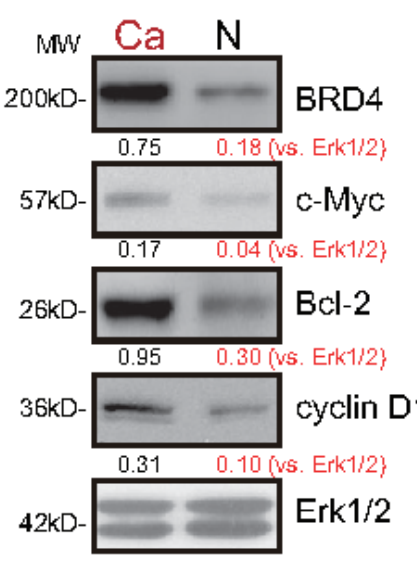

Patient-4
D.

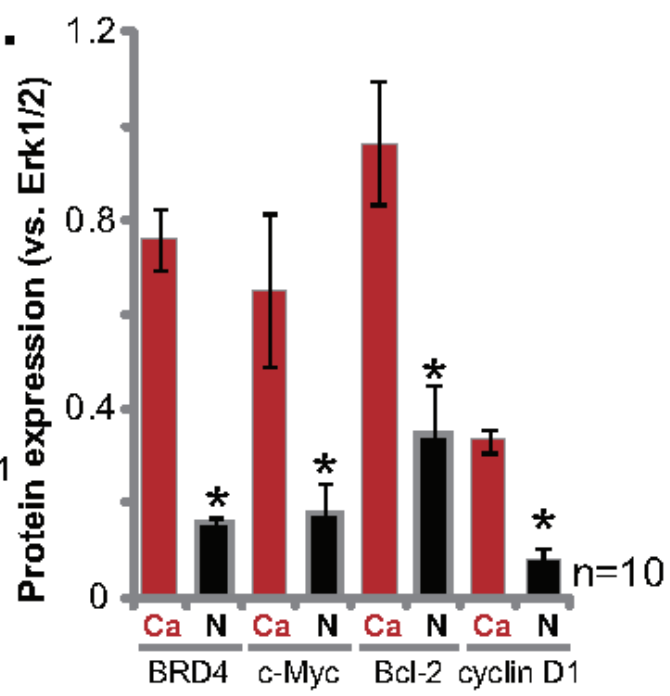

Figure 6

miR-4651 is downregulated in human lung cancer tissues, correlating with upregulation of BRD4 and its target genes. Total RNA and proteins were extracted from a total of ten (10) pairs human NSCLC tissues ("Ca") and surrounding normal lung epithelial tissues $(\mathrm{N}$ "), relative expression of miR-4651 (A), listed mRNAs (B) and proteins ( $C$ and D) were tested by qPCR and Western blotting assays. Expression of listed proteins was quantified, normalized to the loading control Erk1/2 (C). Data were presented as mean \pm standard deviation (SD). *P $<0.05$ vs. "Ca" tissues. The experiments in this figure were repeated five times with similar results obtained. 